\title{
Kaposi's varicelliform eruption following brentuximab vedotin chemotherapy is associated with robust anti-tumor response in an advanced mycosis fungoides patient
}

\author{
Zaw H Phyo ${ }^{1}$, Powell Perng ${ }^{1}$, Sahar Soleimani ${ }^{2}$, Jun Kang ${ }^{1}$, Richard Ambinder ${ }^{3}$ and Sima Rozati ${ }^{*}$ \\ ${ }^{1}$ Department of Dermatology, Johns Hopkins University, Baltimore, MD, USA \\ ${ }^{2}$ Department of Radiology, Johns Hopkins University, Baltimore, MD, USA \\ ${ }^{3}$ Department of Oncology, Johns Hopkins University, Baltimore, MD, USA
}

\begin{abstract}
Kaposi varicelliform eruption (KVE) typically manifests in the setting of underlying chronic inflammatory skin conditions and requires timely intervention with antiviral treatments for favorable morbidities and mortality. In this case presentation, we discuss a 52-year-old woman who developed punched-out erosions and ulcerations involving $50 \%$ of body surface consistent with KVE in the setting of mycosis fungoides (MF). Treatment with acyclovir resulted in temporary, marked regression of preexisting MF plaques in addition to the resolution of KVE. Lastly, we present a review of literature exploring the implication of enhanced immune response to KVE on anti-tumor response in MF patients.
\end{abstract}

\section{Introduction}

Kaposi varicelliform eruption (KVE, also known as eczema herpeticum) refers to a diffuse, viral skin infection which usually manifests in patients with an existing chronic inflammatory skin disease, classically atopic dermatitis. While HSV1 is the main causative agent, infections with coxsackievirus, vaccinia, and varicella have also been implicated [1]. Cutaneous T cell lymphomas (CTCL) belong to a heterogenous group of lymphoid malignancies originating from skinhoming T cells. Mycosis fungoides (MF) is the most common type of CTCL which typically manifests as a combination of patches, plaques and occasionally tumors [2]. Previously reported cases of eczema herpeticum in MF highlight the life-threatening nature of the condition and the importance of timely recognition and treatment [3]. In this report, we discuss the development, treatment course, and subsequent clinical outcome of KVE in a patient previously diagnosed with advanced MF after treatment with brentuximab vedotin chemotherapy.

\section{Case Report}

A 52-year-old African American woman who was recently diagnosed with stage IVA1 MF (T3NxMxB2b) with skin punch biopsy demonstrating no evidence of large cell transformation or follicular tropism with occasional $\mathrm{CD} 30^{+}$cells presented to the infusion center for her second cycle of brentuximab vedotin. In this patient, brentuximab vedotin was chosen as first line therapy given its efficacy in mycosis fungoides irrespective of CD30 level or large cell transformation status [4]. During this visit, she was found to be acutely hypotensive and hypoxic and was promptly admitted to the oncology intensive care unit for respiratory failure and septic shock from fungemia and Corynebacterium bacteremia.

Physical examination revealed punched-out erosions and ulcerations with a scalloped border involving approximately $50 \%$ of body surface and conforming strikingly to the areas of skin involved in MF (Figure 1A-1B). Groups of intact vesicles were appreciated on the forehead, abdomen and flanks with no ocular, oral, or vaginal mucosal involvement. Viral nucleic acid amplification test sampled from the unroofed vesicles on the abdomen were positive for HSV1, consistent with KVE superimposed on MF. The patient was then started on broadspectrum antibiotics, antifungal, and intravenous acyclovir resulting in resolution of bacteremia, fungemia and subsequent slow crusting of vesicles and re-epithelization. At discharge, there was significant regression of prior MF plaques and tumors in parallel to continued repigmentation (Figure 1C) as well as an impressive decrease in size and number of involved lymph nodes compared to baseline (Figure 2A$2 \mathrm{~B})$. However, this anti-tumor response was relatively short-lived with ensuing MF progression after two months.

\section{Discussion}

In this case report, we presented a patient with advanced MF who developed diffuse skin erosions and ulcerations consistent with KVE after her first cycle of brentuximab vedotin treatment. Infection poses a significant risk factor to CTCL patients due to deficits in the skin barrier and immunocompromise attributed to the underlying

${ }^{\star}$ Correspondence to: Sima Rozati, Department of Dermatology, Johns Hopkins University, Baltimore, MD, USA, M.D 601 N Caroline St. Rm 8060B, Baltimore, MD 21297, USA, Tel: 410-955-5933; Fax: 410-502-2309; E-mail: srozati1@jhmi.edu

Key words: kaposi varicelliform eruption, eczema herpeticum, mycosis fungoides, cutaneous T cell lymphoma (CTCL)

Received: March 25, 2021; Accepted: March 29, 2021; Published: March 31, 2021 


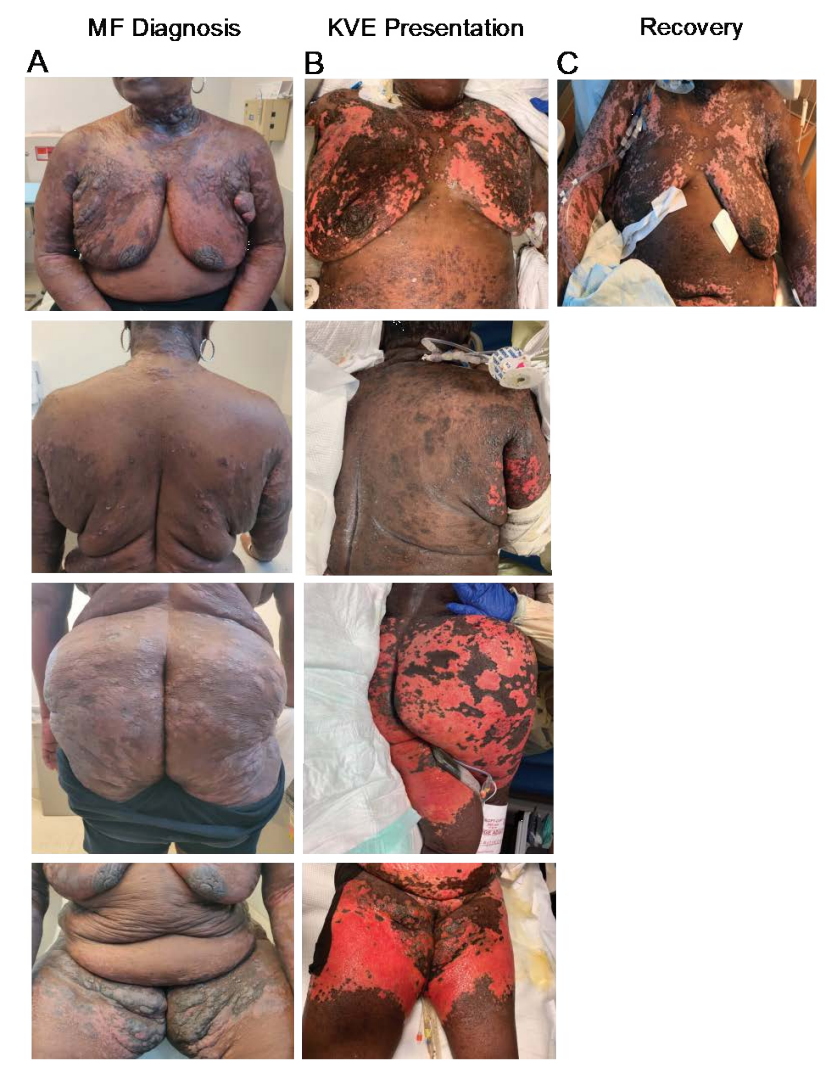

Figure 1. A. Skin plaques and tumors consistent with MF were observed predominantly in breasts, buttocks and thighs at the time of diagnosis; B. Large, eroded plaques in KVE presentation demonstrating high overlap with body surface area involved in MF. C. Recovery from KVE indicated by re-epithelialization and regression of MF plaques and tumors at discharge
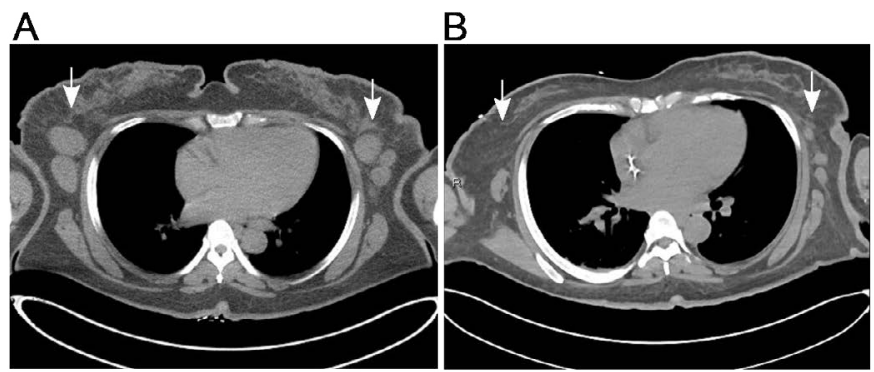

Figure 2. Axial $\mathrm{CT}$ images of the upper chest at the level of axilla demonstrate decrease in the size and number of lymphadenopathy from A, the time of staging to B, two months interval following first Brentuximab vedotin cycle and subsequent KVE infection

malignancy. At the cellular level, deficiency in IFN- $\gamma$ inflammatory cytokine production [5] and aberrant T helper type 2 responses [6] have been implicated as risk factors for KVE in atopic dermatitis patients, although these associations have yet to be confirmed in a larger study and in context of CTCL.

While KVE is classically associated with atopic dermatitis, rare cases have been reported in context of MF. Various treatment modalities in CTCL, including photochemotherapy [3] and skin radiotherapy [7], have been linked to KVE. In this patient, brentuximab-induced necrosis may have been a pathological antecedent which predisposed the patient to superinfection by HSV1 and subsequent fungemia and bacteremia. Timely diagnosis and intervention of KVE are crucial as delayed acyclovir treatment is associated with fatal outcomes [3] and longer hospitalization times [8]. In addition, clinical regression of MF plaques, tumors and lymph nodes following KVE resolution are unlikely to be attributed to brentuximab vedotin treatment as median time to clinical response is 12 weeks in CTCL patients, preceded by 3-4 infusion cycles [9]. Therefore, we postulate that robust inflammatory cytokines release potentiated by HSV1 superinfection may result in enhancement of anti-tumor response. Indeed, intralesional gene transfer of IFN- $\gamma$ and attenuated virus vaccines have been previously investigated for therapeutic use in cutaneous lymphomas given their role in promoting anti-tumor response by enhancing tumor recognition and activating cytotoxic T lymphocytes [10,11].

Our case report highlights the importance of recognizing the clinical characteristics of KVE onset in CTCL patients, as it requires timely diagnosis and treatment. Immunocompromised status in in advanced CTCL patients may predispose involvement of a large body surface area and exacerbate risk for sepsis from secondary translocation of microorganisms, as was the case with this patient. Further, the reconstitution of patient's immune response to HSV1 superinfection resulting in a transient robust antitumor cytotoxic response and significant decrease in tumor burden serves a compelling observation for further development of viral vaccine treatments in CTCL.

\section{References}

1. Ferrari B, Taliercio V, Luna P, Abad M, Larralde M (2015) Kaposi's varicelliform eruption: A case series. Indian Dermatol Online J 6: 399-402. [Crossref]

2. Willemze R, Jaffe ES, Burg G, Cerroni L, Berti E, et al. (2005) WHO-EORTC classification for cutaneous lymphomas. Blood 105: 3768-3785. [Crossref]

3. Segal RJ, Watson W (1978) Kaposi’s Varicelliform Eruption in Mycosis Fungoides Arch Dermatol 114: 1067-1069.

4. Kim YH, Prince HM, Whittaker S, Horwitz SM, Duvic M, et al. (2018) Superior Clinical Benefit of Brentuximab Vedotin in Mycosis Fungoides Versus Physician's Choice Irrespective of CD30 Level or Large Cell Transformation Status in the Phase 3 ALCANZA Study. Blood.

5. Wollenberg A, Wagner M, Günther S, Towarowski A, Tuma E, et al. (2002) Plasmacytoid dendritic cells: A new cutaneous dendritic cell subset with distinct role in inflammatory skin diseases. J Invest Dermatol 119: 1096-1102. [Crossref]

6. Traidl S, Kienlin P, Begemann G, Jing L, Koelle DM, et al. (2018) Patients with atopic dermatitis and history of eczema herpeticum elicit herpes simplex virus-specific type 2 immune responses. J Allergy Clin Immunol 141: 1144-1147.e5.

7. Smith BD, Son CB, Wilson LD (2003) Disseminated Herpes Simplex after Total Skin Electron Beam Radiotherapy for Mycosis Fungoides. J R Soc Med 96: 500-501. [Crossref]

8. Aronson PL, Yan AC, Mittal MK, Mohamad Z, Shah SS (2011) Delayed acyclovir and outcomes of children hospitalized with eczema herpeticum. Pediatrics 128: 1161-1167. [Crossref]

9. Kim YH, Tavallaee M, Sundram U, Salva KA, Wood GS, Li S, et al. Phase II investigator-initiated study of brentuximab vedotin in mycosis fungoides and Sézary syndrome with variable CD30 expression level: A multi-institution collaborative project. J Clin Oncol 33: 3750-3758. [Crossref]

10. Dunn GP, Koebel CM, Schreiber RD (2006) Interferons, immunity and cancer immunoediting. Nat Rev Immunol 6: 836-848. [Crossref]

11. Dummer R, Eichmüller S, Gellrich S, Assaf C, Dreno B, et al. (2010) Phase II clinical trial of intratumoral application of TG1042 (Adenovirus-interferon- $\gamma$ ) in patients with advanced cutaneous T-cell lymphomas and multilesional cutaneous B-cell lymphomas. Mol Ther 18: 1244-1247. [Crossref]

Copyright: (C2021 Phyo ZH. This is an open-access article distributed under the terms of the Creative Commons Attribution License, which permits unrestricted use, distribution, and reproduction in any medium, provided the original author and source are credited. 